\title{
Threat and Risk Management Framework for eHealth IoT Applications
}

\author{
Oleksandr Tomashchuk \\ oleksandr.tomashchuk@philips.com \\ Philips Research \\ Eindhoven, Netherlands \\ imec-DistriNet, KU Leuven \\ Heverlee, Belgium
}

\begin{abstract}
The impact of the Internet of Things (IoT) on the modern industrial and commercial systems is hard to be underestimated. Almost every domain favours from the benefits that IoT brings, and healthcare does not make an exception. This is also clearly demonstrated by a widespread adoption of eHealth systems that often arise from software product lines. Nevertheless, the benefits that IoT brings come together with new threats and risks.

An eHealth system that processes many types of sensitive data sets the context for this thesis. Security and privacy gain crucial importance for successful operation and broad user acceptance of the system because of the properties of the data flows that it initiates and operates. However, due to a large number of feature combinations that originate from the software product line nature of the eHealth system in question, a combinatorial explosion of relevant configurations makes reaching security and privacy goals more difficult. Furthermore, another combinatorial explosion of threats and corresponding mitigation strategies for every configuration complicates the situation even further. Nonetheless, configurations that meet specific risk budgets need to be in place.

Within this thesis, a new threat and risk management (TRM) framework will be provided. It is based on STRIDE and LINDDUN methodologies, and it will overcome existing limitations by employing components on feature space modelling, risk-driven scoring, configuration decision support, and regulatory compliance. Research outcomes that have been reached so far show promising developments on the vital framework components.
\end{abstract}

\section{CCS CONCEPTS}

-Security and privacy $\rightarrow$ Mobile platform security; Data anonymization and sanitization; Usability in security and privacy; Privacy protections; Mobile and wireless security; Information flow control; - Software and its engineering $\rightarrow$ Software product lines.

Permission to make digital or hard copies of all or part of this work for personal or classroom use is granted without fee provided that copies are not made or distributed for profit or commercial advantage and that copies bear this notice and the full citation on the first page. Copyrights for components of this work owned by others than ACM must be honored. Abstracting with credit is permitted. To copy otherwise, or republish, to post on servers or to redistribute to lists, requires prior specific permission and/or a fee. Request permissions from permissions@acm.org.

SPLC '20, October 19-23, 2020, MONTREAL, QC, Canada

(C) 2020 Association for Computing Machinery.

ACM ISBN 978-1-4503-7570-2/20/10 . \$15.00

https://doi.org/10.1145/3382026.3431250

\section{KEYWORDS}

security, privacy, framework, threats, risks, de-identification

ACM Reference Format:

Oleksandr Tomashchuk. 2020. Threat and Risk Management Framework for eHealth IoT Applications. In 24th ACM International Systems and Software Product Line Conference - Volume B (SPLC '20), October 19-23, 2020, MONTREAL, QC, Canada. ACM, New York, NY, USA, 7 pages. https://doi.org/10. $1145 / 3382026.3431250$

\section{INTRODUCTION AND MOTIVATION}

Nowadays, as envisioned by some researchers, the Internet is transforming into the Internet of Things [20]. Despite many expectations, it is not becoming a brand new class of systems. As foreseen, it arises in the form of a next step in the evolution of the Internet, and it progressively extends existing ICT systems and provides additional functionalities [22].

IoT increases its influence on almost every sphere of human activity and brings improvements in efficiency and effectiveness of many processes. The healthcare domain makes no exception to this [25]. The eHealth applications supported by IoT technology that vary from real-time location systems of staff and items in hospitals to continuous monitoring of patients in a home environment with the usage of smart patches start gaining acceptance among healthcare providers.

eHealth IoT systems that customers face with nowadays often arise from well-established software product lines (SPLs). The usage of the latter is not only driven by time and cost reductions that arise from components reuse, but also by the fact that applying SPLs enable more straightforward product evolution and increase scalability which is very important in the healthcare sector.

Improvements that are driven by IoT and SPLs come at a price of new threats and risks which also become broadly imposed. Each eHealth application case involves its inherent design challenges, and -given the nature of the involved data- these systems impose even more substantial threats and risks. Considering the complexity of eHealth IoT systems and a large amount of security and privacy trade-offs related to them, it is a great challenge to design solutions that meet user and business needs while preserving security and privacy. The absence of such preservation causes significant financial and reputational losses [5].

This thesis is done in the context of the HEART project [1], which is aimed at the development of a health-related activity recognition system. This system is expected to rely on a software product line, that has to analyse sleep, nutrition, body parameters, and other kinds of relevant sensitive data. Its architecture (which is under 
development) includes numerous features, combination of which lead to a combinatorial explosion. At the current stage, more than 6000 valid architecture configurations have been identified, and this number is expected to grow. All the configurations and underlying features have to be analysed for providing security and privacy at a satisfactory level.

Among all the existing methodologies, using STRIDE [16] and LINDDUN [10] proved to be a decent approach towards security and privacy threat modelling [26]. Nevertheless, these methodologies were designed for assessing a single configuration of an architecture represented in the form of a data flow diagram. Therefore, STRIDE and LINDDUN cannot provide management of the complete security and privacy configuration scope of complex architectures and ecosystems. Furthermore, every threat assessment of a relatively large architecture that is done with the usage of these methodologies ends up in an additional combinatorial explosion of threats and corresponding mitigation strategies.

In this thesis, an efficient approach towards management of threats and risks is envisioned to be reached by combining the methodologies in question with specific supporting components under a threat and risk management (TRM) framework. To overcome existing limitations, the framework will include threat analysis of configurations and underlying features, assessment of consequent risks, corresponding scoring, and finding most appropriate configuration for a given set up of an eHealth IoT system. The main novelty will come within the components on feature space modelling that is required as a pre-requisite for threat assessment, risk-driven scoring, configuration decision support, and regulatory compliance.

\section{IDENTIFIED GAP AND RESEARCH QUESTION}

Threat modelling is an approach which significantly contributes to improving security and privacy of systems through identifying threats, which in their turn, enable the development of corresponding mitigations. However, complex systems and ecosystems often have vast degrees of variability in terms of architectural components and features that are system-specific, as well in terms of appropriate security/privacy safeguards. This results in combinatorial explosions, which are also exacerbated by the critical nature of eHealth applications. The entire space of possible combinations in such explosions is often not taken into account by developers due to the high complexity of such a task. However, efficient solutions for finding suitable configurations should be in place.

Efficiency in questions related to security and privacy often comes along with concepts of certain budgets. In this case, risk, security, and privacy budgets have to be considered. Risk budget is a certain amount of risk that a stakeholder is willing to take for a specific solution. Security and privacy budgets are components of the risk budget, and they represent the amount of risk that keeps a trade-off value under a certain threshold. For example, in case of a privacy trade-off between data utility and disclosure risk (Figure 1), the part of the risk function that remains under the threshold is included within the privacy budget. Operating risk, security, and privacy budgets contribute to defining suitability of a configuration of features and mitigations.

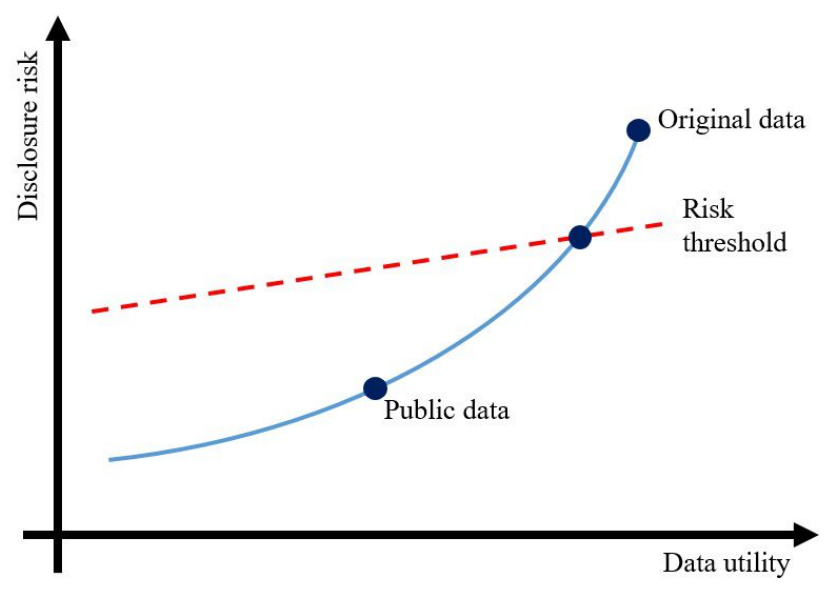

Figure 1: Data utility vs. disclosure risk (from: [17])

Assessment of systems from the security and privacy points of view is done with the usage of certain methodologies. In the security sub-domain, the de-facto standard methodology is STRIDE [15]. In terms of privacy, LINDDUN [10] and its modifications are gaining similar momentum [11]. Both methodologies act upon Data Flow Diagrams (DFDs) as system representation, which makes them complementary. The efficiency of their synergy in terms of security and privacy threat modelling is proven by the SPARTA tool introduced in [28]. Since STRIDE and LINDDUN miss on risk assessment [7], SPARTA tries to enrich them with FAIR [13] risk analysis, but it does not provide means for managing variability and providing scoring for system configurations. There were some attempts to cover architectural trade-offs and variant explosions (e.g. [27]), but security and privacy parts were missing. In general, the main shortcomings of the existing state of the art are:

- threat assessment is performed for a single system configuration;

- feature and threat variabilities as well as their combinatorial explosions are not taken into account;

- security and privacy trade-offs are not considered alongside risk budgets.

Considering the above, the research question can be formulated as follows:

RQ. How to identify configurations of features and mitigations that satisfy certain risk budget, through systematic exploration of their configuration space - even when these incur combinatorial explosions?

\section{RESEARCH METHODOLOGY AND APPROACH}

The research on the identified question is conducted in the specific context of the HEART project [1], which is aimed at the development of an integrated platform for IoT-based eHealth services based on a personalized approach of coaching algorithms that are tailored to individual needs and targeted at the European and Chinese markets. The main industrially-driven contextual use cases foreseen within the platform involve analysis of (i) user's body parameters, (ii) sleep patterns, and (iii) nutrition behaviours. 
Some of the main challenges that this project faces is efficient protection of personal information and providing the users with an appropriate level of security. Therefore, it is required that the platform should be able to provide sufficient levels of security and privacy, as well as comply with the applicable data protection laws such as the GDPR and the CSL.

Closing the identified gap and answering the corresponding research question is a complex task that will require advancements from multiple sides. However, it is foreseen that the advancements will come under an umbrella-framework. The current proposal for such a framework involves the four stages depicted on Figure 2 and described below:

(1) Modelling feature variability of a certain system. This includes covering a space of all possible features that the system's architecture may possess and generating all valid feature configurations of them.

(2) Performing threat assessments from the security and privacy points of view. Also, during this stage, the extraction of risks from the identified threats takes place. Considering the input from the first stage, the assessment and risk extraction takes place for every valid configuration.

(3) Generating security and privacy scores for every configuration and feature based on specific metrics and the results from the previous stage.

(4) Finding the most appropriate (from both security and privacy points of view) configuration of the architecture that meets a certain risk budget and satisfies minimal requirements.

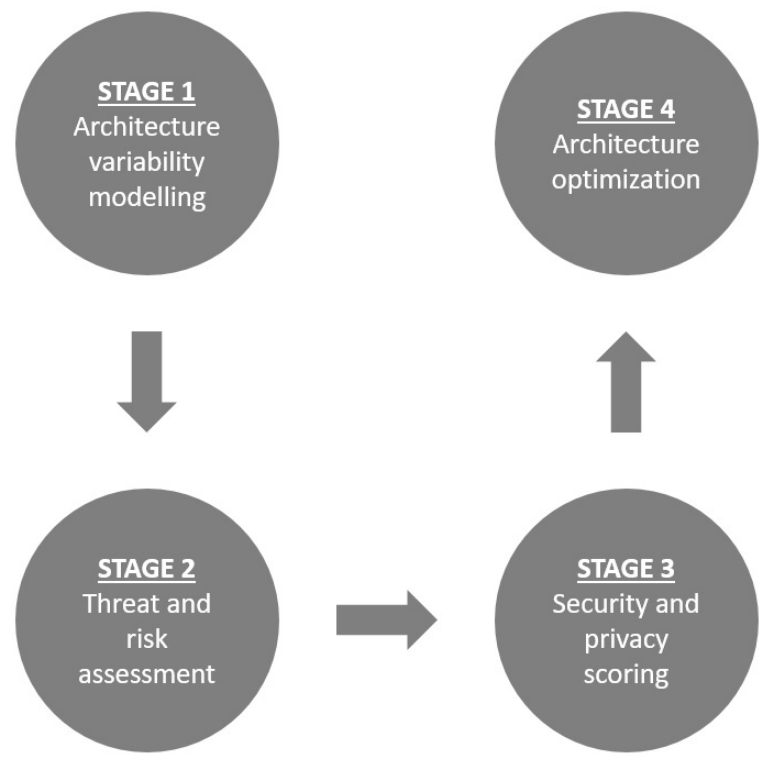

Figure 2: Stages of the framework

The size of the background knowledge related to every stage varies significantly. For example, extensive background on threat and risk assessment results in less efforts that are required for establishing components of the stages 2 and 3. However, due to previously identified gaps, components of the stages 1 and 4 require significant investigation and research efforts. It is envisioned that for making the framework operational, research efforts in the following directions is required:

(1) Research on feature space modelling for IoT solutions,

(2) Research on risk-driven scoring for features and configurations,

(3) Research on selection mechanism of solution configurations that match certain security and privacy budgets,

(4) Research on components that can manage combinatorial explosions of features and mitigations,

(5) Research on minimal regulation-driven security and privacy requirements for IoT solutions.

The envisioned result is a framework that helps architects to take reasonable and appropriate decisions on configurations of features and mitigations for reaching case-specific risk budget while performing full or partial exploration of solution-specific explosions. The main novelty of the framework is a contribution to finding sufficient and representative solutions on system configurations despite facing combinatorial explosions.

One of the main contingencies that may occur while the development of the framework is inoperability between the components that include the former state-of-the-art. For example, difficulties may occur while combining state-of-the-art threat catalogues and mitigation strategies with risk assessment and novel configuration scoring mechanism. Nevertheless, such an issue may be overcome by the development of adaptations to the current risk assessment techniques.

The framework will be validated through a prototype implementation for a reference eHealth IoT architecture which is also considered as an integral part of the research agenda.

\section{PRELIMINARY RESULTS}

The current research efforts are mainly focused on the components of the 1st, 3rd, and 4th stages of the framework. Also, there are ongoing research activities on the modes of the framework's operation for the management of combinatorial explosions as well as the establishment of the eHealth architecture that can be used for the validation purposes. The preliminary research results are discussed in the following subsections.

\subsection{Research on feature space modelling}

The 1st stage of the framework covers the modelling of the space of all possible features that the system's architecture may possess. Whereas the core of the 2nd stage is the DFDs, the result of the feature space modelling should be able to provide sufficient input for the threat assessment. This can be reached through the establishment of a superset DFD that covers all the possible processes, entities, data storages, and data flows. Any valid configuration of a system corresponds to a certain subset DFD that can be derived from the superset one. This derivation can be done with the usage of a feature model and its configurations that are tailored to the superset DFDs in question.

Since contributions to this research direction are considered as work in progress, more details will be provided in the following publications. 


\subsection{Research on selection mechanism of solution configurations that match certain privacy budget}

The result of the threat assessment step in the LINDDUN methodology is a list of privacy threats. Such threats can be neutralised by mitigation strategies that are included in the taxonomy of privacy mitigation strategies which comes as supporting material for the methodology. The majority of the strategies in the taxonomy fall under the scope of de-identification methods. Therefore, to cope with the explosion of possible threat mitigations and satisfying privacy budget, efficient decision support is needed. Such a need is addressed through a data utility-driven benchmark for de-identification methods that has been developed.

In this research, de-identification is considered as a concept of a higher level, which covers both anonymisation [2] and pseudonymisation [31], [3]. The definition of de-identification that is provided in [14] was also selected for establishing a strong terminological basis for the research.

De-identification is implemented by certain methods, aimed at satisfying privacy requirements through reducing the amount of identifying information. There are many de-identification methods, and despite attempts to systematize them ([23], [4], [6], [19]), a support for making decisions on their selections has not been in place.

Considering the inherent nature of de-identification methods and their functional purpose, one can decide on suitability of a method based on data utility loses that it causes [24], as well as the privacy level that it provides within certain privacy models ( $k$-anonymity [30], $l$-diversity [21], $t$-closeness [18], $\beta$-likeness [9], $\delta$-presence [12], $\delta$-disclosure [8], etc.). To support such a decision a benchmark that takes these factors into account was introduced in [33]. The diagram that describes flow-based representation of the proposed benchmark system can be found on Figure 3.

The introduced benchmark implements an exhaustive search for the most appropriate methods and their combinations in terms of two key factors: (i) adherence to the privacy requirements and (ii) data utility loss of the transformed data set. It also implements a two-phased approach: (i) in the expansion phase, candidate methods are generated, and (ii) in the reduction phase, these are filtered based on the privacy requirements (which act as a cut-off) and the data utility score. The benchmark is exhaustive in a way that it applies different methods at an attribute-level and allows combining different methods within the same data set. Furthermore, it systematically iterates over parameter values of the different methods, and thus allows assessing the impact of these parameters. More detailed information on the benchmark, as well as its validation, can be found in [33].

\subsection{Research on minimal regulation-driven security and privacy requirements for IoT solutions}

Regulations are the key drivers of establishing minimum levels of security and privacy for industrial systems. Their interpretations by legal experts lead to obtaining legal requirements. Therefore, considering the HEART context, a list of legal requirements that arise

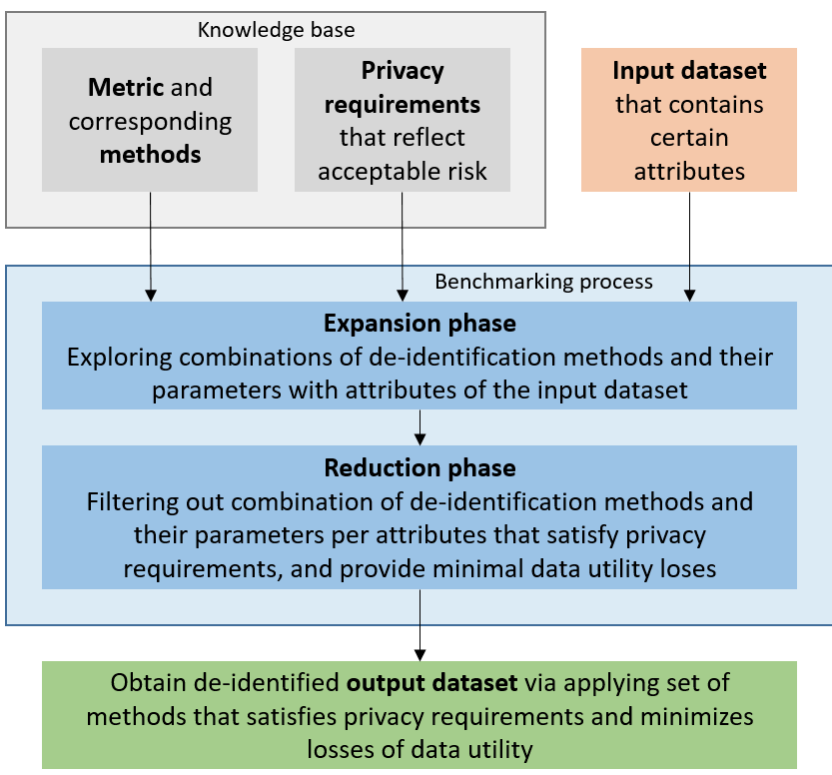

Figure 3: Flow diagram of the data utility-driven benchmark for de-identification methods (from: [33])

from the EU's General Data Protection Regulation (GDPR) [31] and China's Cybersecurity Law (CSL) Standing Committee of the People's Congress 29 was established. This also enabled the translation of the legal requirements into technical ones, that can have a direct influence on the solutions architectures.

Having the sole technical requirements is not solving the challenge of regulatory compliance. Therefore, an exemplar case study of a generic eHealth IoT system was established for gaining practical insights on reaching GDPR and CSL compliance. This allowed to get (i) an overview of the influence of the GDPR and the CSL requirements on data flows in a simplified architecture, and (ii) an extension of the reference eHealth IoT architecture with identified requirements-driven elements for simultaneous compliance.

For the reason of satisfying the technical requirements, concepts of services and centres for data flow diagrams were introduced. These concepts are not positioned as an extension of existing DFD elements (entity, process, data store, data flow, trust boundary), but rather as aggregators. Subsequently, the services are combinations of several processes that perform specific manipulations on data. In their turn, the centres are combinations of services that need to take place for performing necessary data manipulations. An extension with centres and services that correspond to the technical requirements enabled GDPR and CSL compliance for the reference eHealth IoT architecture. A compliant version of such architecture can be found on Figure 5. More inputs regarding the requirements, DFD extensions, and case study can be found in [32].

The processes, services, and centres can also be considered as system features. Therefore, minimal requirements that are reflected by them can be easily integrated into the threat and risk management framework in question. 

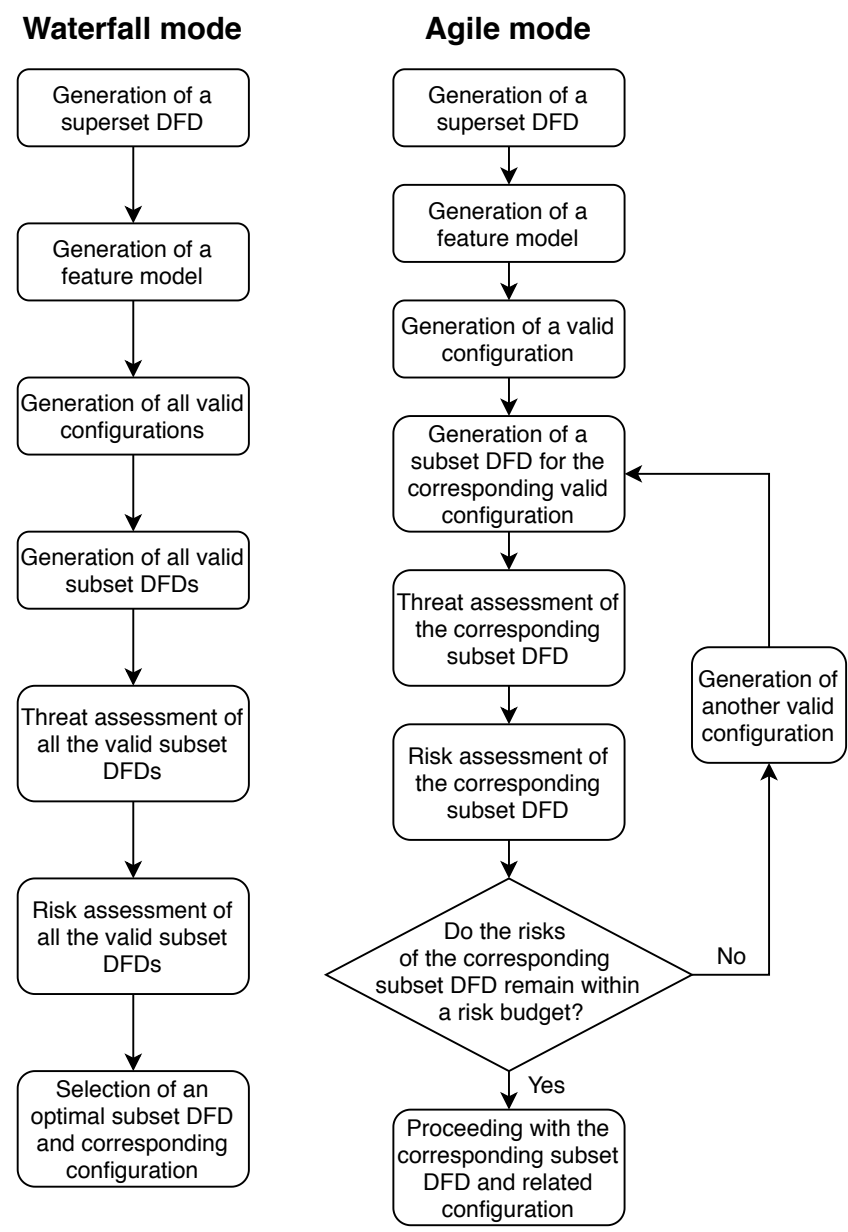

Figure 4: Operation modes of the proposed framework

\subsection{Research on management of combinatorial explosions}

A naive approach to the operation of the framework is based on a step-by-step process that accordingly to the stages includes:

(1) Generation of a superst DFD, feature model, all valid configurations and corresponding subset DFDs,

(2) Threat assessment of all the valid subset DFDs,

(3) Risk assessment of all the valid subset DFDs,

(4) Selection of an optimal subset DFD based on the scoring of features and configurations.

Such a mode of operation can also be called "waterfall" (see Figure 4). As a result of the operation in this mode, the framework can provide an architect with an optimal system configuration in terms of security and privacy. However, the waterfall mode suffers in terms of performance when the amount of features and threats is relatively high. This drawback can be overcome by the so-called "agile" mode of operation (see Figure 4), which does not perform an exhaustive assessment for every valid configuration, but instead selects one and checks its fitting within a risk budget. Nonetheless, the improvement in terms of performance come at a price of precision - the larger is the risk budget, the cheaper (in terms of computational efforts) it can be to find a suitable configuration.

Since research on the operation modes of the framework is considered as work in progress, both of the modes require further investigations. One of the most promising improvement points is an efficient selection of configurations in the agile mode. Such a selection may employ Breadth-First Search (BFS), Depth-First Search (DFS), or other search algorithms. More details on this point will be provided in the following publications.

\subsection{Establishment of the Reference eHealth IoT architecture}

Validation of the framework for eHealth IoT solutions requires a sufficient reference architecture that covers the most significant domain-specific aspects. The reference architecture that was introduced in [32] needs to be further developed, as at the current stages it represents only the high-level details.

The reference eHealth IoT architecture can be enriched through the integration of (i) use case-driven platform features: cloud as well as local analytics (body parameters, sleep, activity, nutrition, mental well-being, environment), third party data sharing, the capability of platform extension by user's smart devices; (ii) security features: encryption, access control and logging, user authentication, data de-identification, backups, GDPR compliance, CSL compliance; as well as other features. More details on the extended reference architecture will be provided in the following publications.

\section{WORK PLAN}

Considering the current research progress that has been highlighted in the previous section, the work plan for the next 18 months consists of research on the following topics:

- Feature space modelling for security and privacy threat assessment

- Risk driven scoring of features and configurations

- Method of identification of system configurations that match certain security budget

- Further investigation on the operation modes of the framework

- Develop a prototype implementation of the framework and apply it to the reference eHealth IoT architecture

- Compare the framework with the relevant threat- and riskanalysis approaches

- Feasibility of delegation of decisions on trade-offs from architects to end users

- Evaluation of opportunities for the usage of the framework outside the eHealth subdomain

\section{ACKNOWLEDGMENTS}

This research is funded by Philips Research and the HEART project (www.heart-itn.eu). This project has received funding from the European Union's Horizon 2020 research and innovation programme under the Marie Skłodowska-Curie grant agreement No 766139. This publication reflects only the authors' view and the REA is not responsible for any use that may be made of the information it contains. 


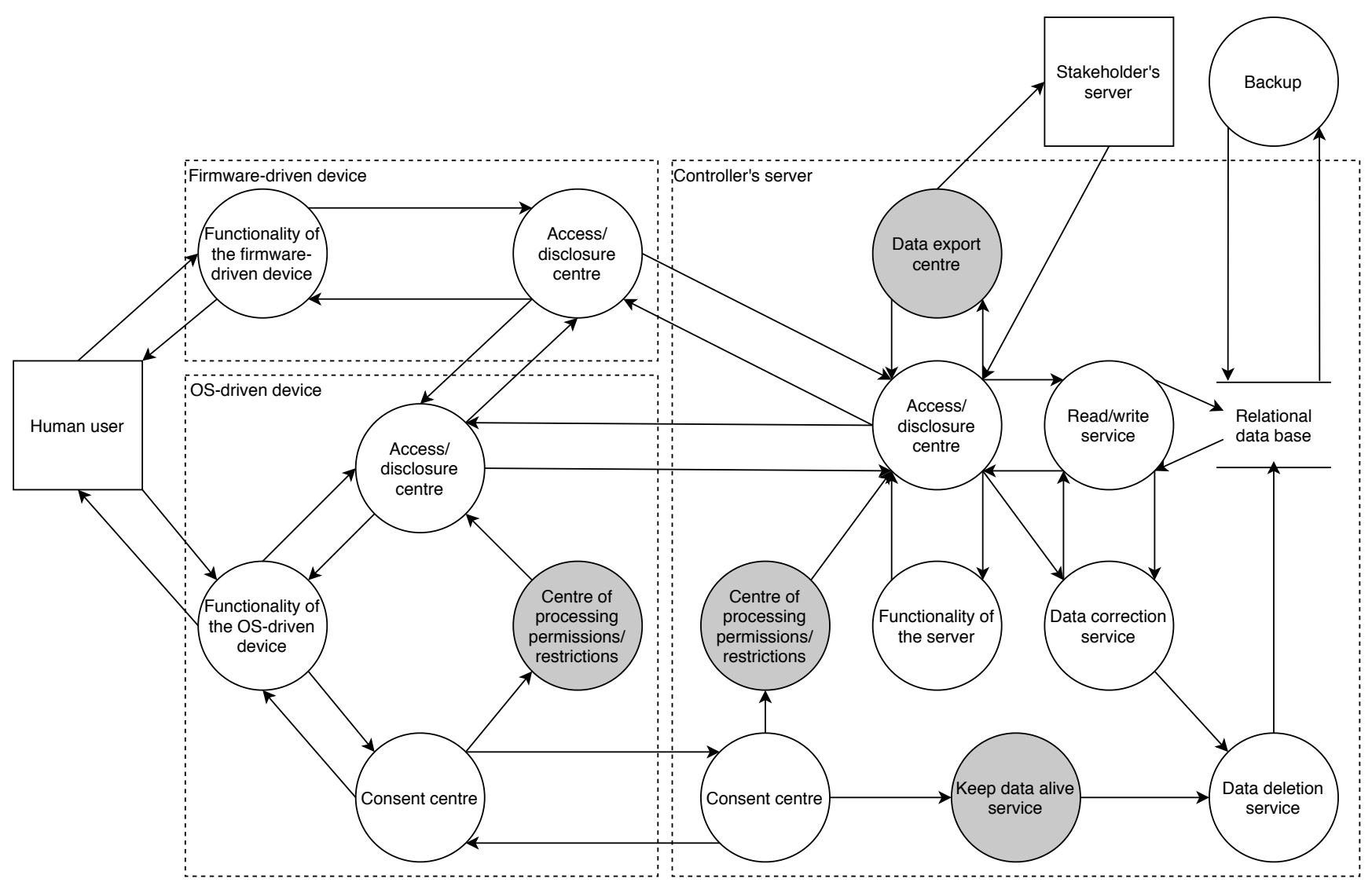

Figure 5: DFD of a reference eHealth IoT architecture extended with the requirements-driven elements. Grey elements are optional for CSL-only compliance. (from: [32])

\section{REFERENCES}

[1] [n. d.]. HEalth related Activity Recognition system based on IoT. http://heart-itn. $\mathrm{eu} /$

[2] 2011. ISO/IEC 29100 Information technology - Security techniques - Privacy framework. (2011).

[3] 2017. ISO 25237 - Health informatics - Pseudonymization. (2017)

[4] 2018. ISO/IEC 20889 - Privacy enhancing data de-identification terminology and classification of techniques. (2018).

[5] Mackenzie Adams. 2017. Big Data and Individual Privacy in the Age of the Internet of Things. Technology Innovation Management Review 7 (2017). Issue 4.

[6] Article 29 Data Protection Working Party. 2014. 0829/14/EN WP216 Opinion 05/2014 on Anonymisation Techniques. https://www.pdpjournals.com/docs/ 88197.pdf. (2014)

[7] Tamas Bisztray and Nils Gruschka. 2019. Privacy Impact Assessment: Comparing Methodologies with a Focus on Practicality. In Proceedings of NordSec 2019. 3-19.

[8] Justin Brickell and Vitaly Shmatikov. 2008. The cost of privacy: destruction of data-mining utility in anonymized data publishing. In Proceedings of the 14th ACM SIGKDD international conference on Knowledge discovery and data mining.

[9] Jianneng Cao and Panagiotis Karras. 2012. Publishing microdata with a robust privacy guarantee. In Proceedings of the VLDB Endowment, vol.5.

[10] Mina Deng, Kim Wuyts, Riccardo Scandariato, Bart Preneel, and Wouter Joosen 2011. A privacy threat analysis framework: supporting the elicitation and fulfillment of privacy requirements. Requirements Engineering fournal 16 (2011) 3-32.

[11] Axel Ekdahl and Lídia Nyman. 2019. A Methodology to Validate Compliance to the GDPR.

[12] Mehmet Ercan Nergiz, Maurizio Atzori, and Chris Clifton. 2007. Hiding the presence of individuals from shared databases. In Proceedings of the 2007 ACM SIGMOD international conference on Management of data.
[13] Jack Freund and Jack Jones. 2014. Measuring and Managing Information Risk: A FAIR Approach. Butterworth-Heinemann, USA.

[14] Simson L Garfinkel. 2015. NIST IR 8053: De-Identification of Personal Information. http://dx.doi.org/10.6028/NIST.IR.8053. (2015).

[15] S. Hernan, S. Lambert, T. Ostwald, and A. Shostack. 2006. Threat Modeling: Uncover Security Design Flaws Using The STRIDE Approach. MSDN Magazine 6 (2006).

[16] Loren Kohnfelder and Praerit Garg. 1999. The threats to our products. Microsoft Interface (April 1999)

[17] Yong Ju Lee and Kyung Ho Lee. 2018. What are the optimum quasi-identifiers to re-identify medical records? In Advanced Communication Technology (ICACT), 2018 20th International Conference on. IEEE, 1025-1033.

[18] Ninghui Li, Tiancheng Li, and Suresh Venkatasubramanian. 2007. t-Closeness: Privacy Beyond k-Anonymity and l-Diversity. (2007).

[19] Konstantinos Limniotis and Marit Hansen. 2018. Recommendations on shaping technology according to GDPR provisions - An overview on data pseudonymisation. https://www.enisa.europa.eu/publications/ recommendations-on-shaping-technology-according-to-gdpr-provisions. (2018).

[20] Lu Tan and Neng Wang. 2010. Future internet: The Internet of Things. In 2010 3rd International Conference on Advanced Computer Theory and Engineering(ICACTE), Vol. 5. V5-376-V5-380.

[21] A. Machanavajihala, J. Gehrke, and D. Kifer. 2006. L-diversity: privacy beyond $\mathrm{k}$-anonymity. In 22nd International Conference on Data Engineering (ICDE'06). IEEE.

[22] Daniele Miorandi, Sabrina Sicari, Francesco De Pellegrini, and Imrich Chlamtac. 2012. Internet of things: Vision, applications and research challenges. Ad Hoc Networks 10 (2012), 1497-1516. Issue 7

[23] Gregory S Nelson. 2015. Practical implications of sharing data: a primer on data privacy, anonymization, and de-identification. In SAS Global Forum Proceedings. 
[24] Benjamin Podgursky. 2011. Practical k-anonymity on large datasets. Master's thesis. Vanderbilt University, Nashville, Tennessee.

[25] Y. A. Qadri, A. Nauman, Y. B. Zikria, A. V. Vasilakos, and S. W. Kim. 2020. The Future of Healthcare Internet of Things: A Survey of Emerging Technologies. IEEE Communications Surveys Tutorials 22, 2 (2020), 1121-1167.

[26] Adam Shostack. 2014. Threat Modeling: Designing for Security (1st ed.). Wiley Publishing.

[27] Laurens Sion, Dimitri Landuyt, Wouter Joosen, and Gjalt Jong. 2016. Systematic quality trade-off support in the software product-line configuration process. 164-173. https://doi.org/10.1145/2934466.2934481

[28] L. Sion, D. Van Landuyt, K. Yskout, and W. Joosen. 2018. SPARTA: Security Privacy Architecture Through Risk-Driven Threat Assessment. In 2018 IEEE International Conference on Software Architecture Companion (ICSA-C). 89-92.

[29] Standing Committee of the People's Congress. 2016. Cybersecurity Law. (2016).
[30] Latanya Sweeney and Pierangela Samarati. 1999. Protecting Privacy when Disclosing Information: k-Anonymity and Its Enforcement through Generalization and Suppression. (1999).

[31] The European Parliament and the Council of the European Union. 2016. Regulation (EU) 2016/679 General Data Protection Regulation. Official Journal of the European Union L119 (May 2016), 1-88. http://eur-lex.europa.eu/legal-content/ EN/TXT/?uri=OJ:L:2016:119:TOC

[32] Oleksandr Tomashchuk, Yuan Li, Dimitri Van Landuyt, and Wouter Joosen. 2020. Operationalization of privacy and security requirements for eHealth IoT applications in the context of GDPR and CSL. In Privacy Technologies and Policy.

[33] Oleksandr Tomashchuk, Dimitri Van Landuyt, Daniel Pletea, Kim Wuyts, and Wouter Joosen. 2019. A data utility-driven benchmark for de-identification methods. In TrustBus 2019: Trust, Privacy and Security in Digital Business. 63-77. 